\title{
Study on the transfer probability density function of a class of stochastic differential dynamical systems
}

\author{
Dongwei Huang ${ }^{1}$, Lijiao $\mathrm{Cai}^{1}$, Yongfeng $\mathrm{Guo}^{1}$, and Jianguo Tan ${ }^{1}$ \\ ${ }^{1}$ Tiangong University
}

May 5, 2020

\begin{abstract}
In this paper, the solution and validity of the transfer probability density function for a stochastic dynamical system excited by white Gaussian noise are discussed. Based on the exponential polynomial closure method, not only the numerical solution of FPK equation is accepted, but also the validity of the method is shown from different views. On the one hand, the exact solution expression of the stationary transition probability density of some kind of system is received and its error compared with the numerical solution is analyzed. On the other hand, by establishing a kind of potential function to observe the stable region of the state variables in probabilistic sense, it is found that the stable region of the state variables determined by the potential function is highly consistent with the stable region determined by the stationary transition probability density function after long-term observation.
\end{abstract}

\section{Hosted file}

paper2,(2).pdf available at https://authorea.com/users/303861/articles/434102-study-on-thetransfer-probability-density-function-of-a-class-of-stochastic-differential-dynamicalsystems

\section{Hosted file}

paper2,(2).tex available at https://authorea.com/users/303861/articles/434102-study-on-thetransfer-probability-density-function-of-a-class-of-stochastic-differential-dynamicalsystems 
figures/tu1/tu1-eps-converted-to.pdf 
figures/tu2/tu2-eps-converted-to.pdf 
figures/tu3/tu3-eps-converted-to.pdf 
figures/tu5/tu5-eps-converted-to.pdf 
figures/tu4/tu4-eps-converted-to.pdf 
figures/tu6/tu6-eps-converted-to.pdf 
figures/tu7/tu7-eps-converted-to.pdf 
figures/tu8/tu8-eps-converted-to.pdf 
figures/tu9/tu9-eps-converted-to.pdf 
figures/tu10/tu10-eps-converted-to.pdf 